ISSN 1995-5464 (Print); ISSN 2408-9524 (Online)

DOI: $10.16931 / 1995-5464.2019111-16$

Конфокальная лазерная эндомикроскопия в Аиагностике заболеваний внепеченочных желчных протоков

\author{
Солодинина Е.Н. ${ }^{1}$, Фомичева Н.В. ${ }^{1 *}$, Ульянов Д.Н. ${ }^{2}$ \\ ${ }^{1}$ ФГБУ “Центральная клиническая больница с поликлиникой” УДП РФ; 121359, Москва, \\ ул. Маршала Тимошенко, д. 15, Российская Федерация \\ ${ }^{2}$ European Medical Center; 129090, Москва, ул. Щепкина, д. 35, Российская Федерация
}

\begin{abstract}
Цель. Оценить информативность конфокальной лазерной эндомикроскопии с прицельной биопсией в верификации характера стриктур внепеченочных желчных протоков.

Материал и методы. В исследование включили 28 пациентов со стриктурами внепеченочных желчных протоков. Ретроградное вмешательство дополняли конфокальной лазерной эндомикроскопией с последующей прицельной биопсией. Результаты лазерной эндомикроскопии и биопсии сравнивали с окончательным диагнозом после операции и морфологического исследования, результатами наблюдения за пациентами в течение 1-4 лет при проведении эндоскопического лечения по поводу доброкачественных заболеваний.

Результаты. Диагностическая чувствительность, диагностическая специфичность и общая точность метода в дифференциальной диагностике стриктур общего желчного протока составили 91,7, 93,7 и 92,8\% соответственно. Осложнения отмечены в $1(3,6 \%)$ наблюдении - острый отечный панкреатит.

Заключение. Конфокальная лазерная эндомикроскопия является новым методом, позволяющим прижизненно оценить изменения слизистой оболочки на микроскопическом уровне. Несмотря на техническую сложность, применение метода не сопровождается увеличением частоты осложнений и соответственно не имеет противопоказаний, отличных от ЭРХПГ.
\end{abstract}

Ключевые слова: желчные протоки, стриктура, ЭРХПГ, конфокальная лазерная эндомикроскопия, холангиоскопия. Ссылка для цитирования: Солодинина Е.Н., Фомичева Н.В., Ульянов Д.Н. Конфокальная лазерная эндомикроскопия в диагностике заболеваний внепеченочных желчных протоков. Анналы хирургической гепатологии. $2019 ; 24$ (1): 11-16. DOI: $10.16931 / 1995-5464.2019111-16$.

Авторы подтверждают отсутствие конфликтов интересов.

\title{
Confocal laser endomicroscopy in the diagnosis of extrahepatic bile duct diseases
}

\author{
Solodinina E.N. ${ }^{1}$, Fomicheva N.V. ${ }^{1 *}$, Ulyanov D.N. ${ }^{2}$ \\ ${ }^{1}$ Central Clinical Hospital with a Polyclinic of Presidential Administration of the Russian Federation; \\ 15, Marshala Timoshenko str., Moscow, 121359, Russian Federation \\ ${ }^{2}$ European Medical Center; 35, Schepkina str., Moscow, 129090, Russian Federation
}

\begin{abstract}
Aim. To evaluate information content of confocal laser endomicroscopy with targeted biopsy in verifying etiology of extrahepatic bile duct strictures.

Material and methods. There were 28 patients with extrahepatic bile duct strictures who underwent retrograde intervention with confocal laser endomicroscopy and targeted biopsy. Data of confocal laser endomicroscopy and biopsy were compared with final postoperative and histological diagnosis. Follow-up within 1-4 years after endoscopic treatment was also considered.

Results. Diagnostic sensitivity, specificity and overall accuracy of the method in differential diagnosis of common bile duct strictures were $91.7 \%, 93.7 \%$ and $92.8 \%$, respectively. Complication (acute edematous pancreatitis) occurred in $1(3.6 \%)$ case.

Conclusion. Confocal laser endomicroscopy is new effective method for in vivo microscopic assessment of mucous membrane. Despite technical complexity, the method is not associated with advanced morbidity and, accordingly, has no additional contraindications in comparison with ERCP.
\end{abstract}

Keywords: bile ducts, stricture, ERCP, confocal laser endomicroscopy, cholangioscopy.

For citation: Solodinina E.N., Fomicheva N.V., Ulyanov D.N. Confocal laser endomicroscopy in the diagnosis of extrahepatic bile duct diseases. Annaly khirurgicheskoy gepatologii = Annals of HPB surgery. 2019; 24 (1): 11-16. (In Russian).

DOI: 10.16931/1995-5464.2019111-16.

There is no conflict of interests. 


\section{Введение}

Рост числа пациентов со стриктурами внепеченочных желчных протоков различного генеза происходит на фоне увеличения числа пациентов со злокачественными новообразованиями органов гепатопанкреатодуоденальной зоны (ГПДЗ), составляющими около $15 \%$ всех злокачественных опухолей пищеварительного тракта [1]. Определение этиологии стеноза является ключевым для выбора хирургической тактики. Широко применяемые методы лучевой диагностики позволяют определить природу стеноза только в 70\% наблюдений [2, 3], что требует разработки методов, обладающих более высокой эффективностью. В последнее время в мире все чаще применяют эндоскопическую ретроградную (или интраоперационную антеградную) холедохоскопию с внутрипротоковой конфокальной лазерной эндомикроскопией (КЛЭМ). Метод позволяет получить прижизненную гистопатологическую картину при различных патологических изменениях желчных и панкреатических протоков, а также выполнить прицельный забор материала для морфологического исследования. К настоящему времени зарубежными авторами опубликованы лишь отдельные работы, посвященные холангиоскопии и КЛЭМ при заболеваниях органов ГПДЗ. Это трудоемкие и дорогостоящие методы, но порой только их сочетание позволяет верифицировать диагноз, что особенно актуально в ранней диагностике злокачественных новообразований внепеченочных желчных протоков.

Цель исследования - оценить информативность конфокальной лазерной микроскопии с прицельной биопсией в верификации характера стриктур внепеченочных желчных протоков.

\section{Материал и методы}

Попытка КЛЭМ предпринята у 30 пациентов, однако технически успешной процедура оказалась в 28 наблюдениях. Таким образом, в исследование включено 28 пациентов со стриктурой внепеченочных желчных протоков опухолевого и неопухолевого генеза. Вмешательство выполняла бригада из двух врачей, опыт одного из них составляет более 300 ЭРХПГ. Всем больным выполняли ретроградное вмешательство (ЭРХПГ) при помощи дуоденоскопа TJF 160VR с широкой папиллосфинктеротомией и при необходимости баллонной дилатацией устья общего желчного протока (ОЖП). Холангиоскопию выполняли ультратонким аппаратом Olympus GIF-N180, который проводили в желчные протоки через рот после извлечения дуоденоскопа. Для проведения внутрипротоковой КЛЭМ применяли систему Cellvizio (Mauna Kea Technologies, Франция). Компоненты системы: конфокальный лазерный сканирующий блок F-400 (длина волны 488 нм), компьютер со специальным программным обеспечением для получения изображений, зонд Cholangioflex, дисплей, педаль дистанционного управления.

Зонд, состоящий из тысяч оптических волокон, передает сканирующий лазерный луч в зону наблюдения и фиксирует флуоресцентный свет, отражаемый тканью. Через инструментальный канал зонд подводили к патологически измененному участку слизистой. Гибкая структура зонда позволяет эндоскопу находиться почти в любой анатомической конфигурации. Получаемые изображения подвергаются сканированию со скоростью 12 изображений в секунду, что создает эффект видеофильма. Для получения изображений применяли контрастный препарат - $10 \%$ раствор флуоресцеина натрия, который вводили внутривенно через периферический катетер. Исследование можно начинать через 30 спосле введения препарата. Флуоресцеин не окрашивает клеточные ядра и цитоплазму, накапливаясь в собственной пластинке слизистой. Сканирование продолжали до тех пор, пока не получали изображения морфологических структур. Проводили приблизительную оценку их морфологической принадлежности in vivo в соответствии с эталонными изображениями в руководствах по КЛЭМ. При анализе конфокальной эндомикроскопической картины следует учитывать, что получаемые изображения отличаются от традиционных гистологических препаратов. Ориентация конфокальных оптических срезов имеет горизонтальное направление и скорее оценивает микроархитектонику клеток и сосудов, чем вертикальную дифференцировку слоев, как при традиционном гистологическом срезе [4].

Получаемые во время исследования видеозаписи и отдельные изображения микрососудистого русла и рельефа слизистой внепеченочных желчных протоков сохраняли в цифровом виде для дальнейшего ретроспективного анализа. После КЛЭМ выполняли забор материала для гистологического исследования прицельно из наиболее измененных участков щипцами, которые также проводили через инструментальный канал эндоскопа.

Полученные при КЛЭМ и прицельной биопсии результаты сравнивали с окончательным морфологическим диагнозом.

\section{Результаты}

Среди 28 пациентов было 16 (57,2\%) мужчин и $12(42,8 \%)$ женщин. Средний возраст составил 66 лет. В возрастной группе от 31 до 60 лет было $1(3,6 \%)$ мужчин, в возрастной группе от 61 до 74 лет - $10(35,7 \%)$ мужчин и $6(21,4 \%)$ женщин, в возрасте 75-90 лет было $5(17,9 \%)$ мужчин и $6(21,4 \%)$ женщин. 
Всем пациентам выполняли лабораторные исследования, УЗИ брюшной полости, КТ и (или) МРТ. Лечебные вмешательства выполнены всем пациентам: 15 больным выполнили эндоскопические внутрипросветные вмешательства, в том числе паллиативное стентирование при опухолевом стенозе, 13 - традиционные хирургические вмешательства. Двое больных умерли от прогрессирования основного онкологического заболевания в ближайшие сроки после обследования. Окончательный диагноз установлен во всех наблюдениях. Критерием окончательного диагноза при стенозе злокачественного генеза стали результаты интраоперационной ревизии с биопсией или исследованием удаленной опухоли $(n=13)$, а также секционного материала $(n=2)$. При рубцовом стенозе пациентам выполняли этапное эндоскопическое стентирование пластиковыми стентами. Критерием окончательного диагноза в этих ситуациях считали динамическое наблюдение на протяжении 1-4 лет, при котором не выявили опухоли желчных протоков $(n=14)$.

Доброкачественные заболевания выявлены у 14 (50\%) больных: стриктура желчного протока после холецистэктомии - у 4 (14,3\%), холедохолитиаз - у 8 (28,6\%), первичный склерозирующий холангит - у $2(7,1 \%)$. Злокачественные заболевания диагностированы также у 14 (50\%) больных: протоковая аденокарцинома головки поджелудочной железы - у 11 (39,3\%), холангиокарцинома - у 3 (10,7\%).

Эндоскопическая ретроградная холедохоскопия с КЛЭМ выполнена всем пациентам. При исследовании желчных протоков акцент делали на поиске и оценке размеров капилляров и структуры капиллярной сети. В норме ОЖП покрыт однослойным эпителием, под которым располагается подслизистый слой, создающий эндомикроскопическую картину, представленную выраженной, сложной сетью коллагеновых волокон и лимфатических синусов с ретикулярным расположением. Кровеносные капилляры располагаются на разной глубине подслизистого слоя и представлены тонкими и регулярными светло-серыми полосами (рис. 1). При опухолях в результате неоангиогенеза нормальная сетчатая микроструктура нарушается, замещаясь утолщенными малоизвитыми сосудами, в которых иногда можно проследить кровоток (рис. 2).

Поскольку КЛЭМ не заменяет традиционного гистологического исследования материалов биопсии, последнюю выполняли в самом конце процедуры, поскольку даже незначительное поступление крови может сделать невозможным адекватный осмотр при КЛЭМ. При помощи комбинированного метода диагностики злокачественный характер стриктуры ОЖП заподозрен у 12 пациентов, у 11 (39,3\%) диагноз подтвержден гистологически (истинно положительный
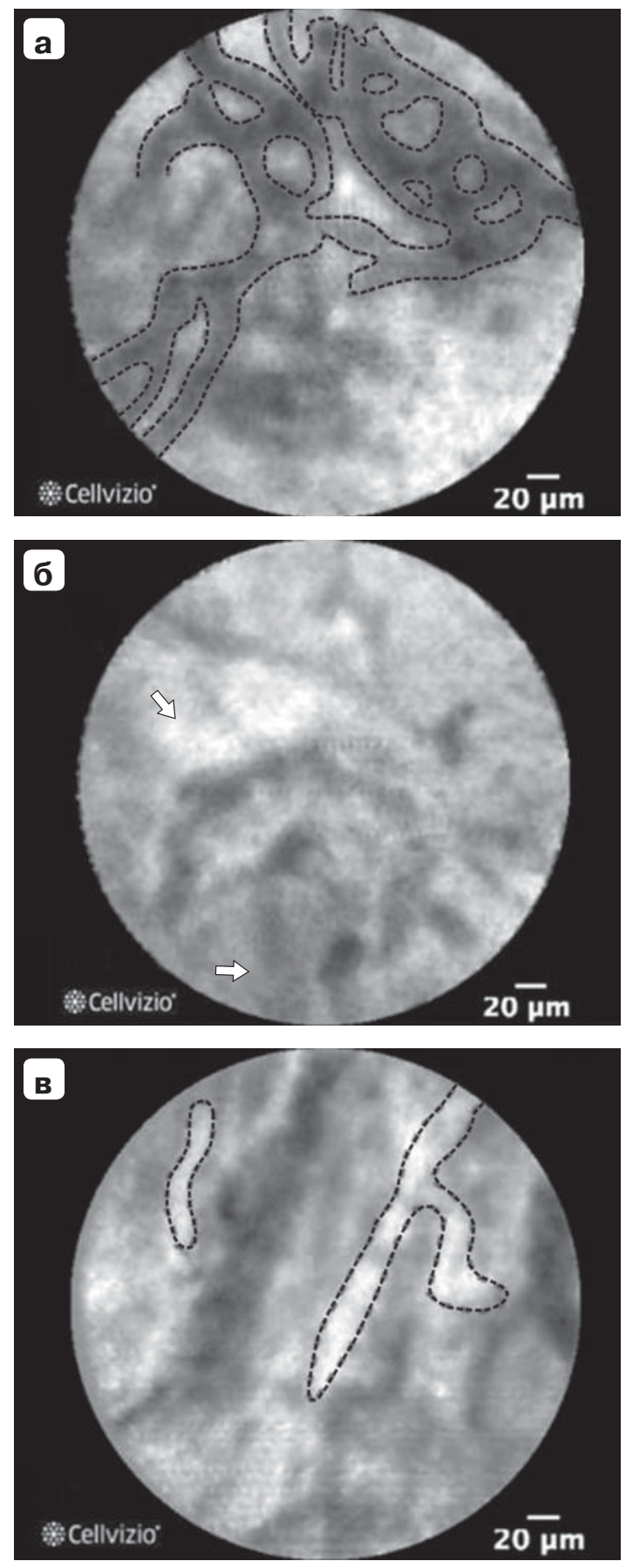

Рис 1. Эндоскопическое микрофото. Конфокальное изображение слизистой оболочки ОЖП в норме: а - сеть коллагеновых волокон в виде тонких темных полосок толщиной менее 20 микрон; б - светло-серый, гомогенный фон; в - кровеносные сосуды толщиной менее 20 микрон.

Fig. 1. Endoscopic photo. Confocal image of normal mucosa of common bile duct: a - collagen fibers network in the form of thin dark strips with a thickness of less than 20 microns; b - light gray, homogeneous background; c - blood vessels with a thickness of less than 20 microns.

результат). При исследовании операционного материала в $1(3,6 \%)$ наблюдении диагноз холангиокарциномы был опровергнут (ложноположительный результат). Ложноотрицатель- 

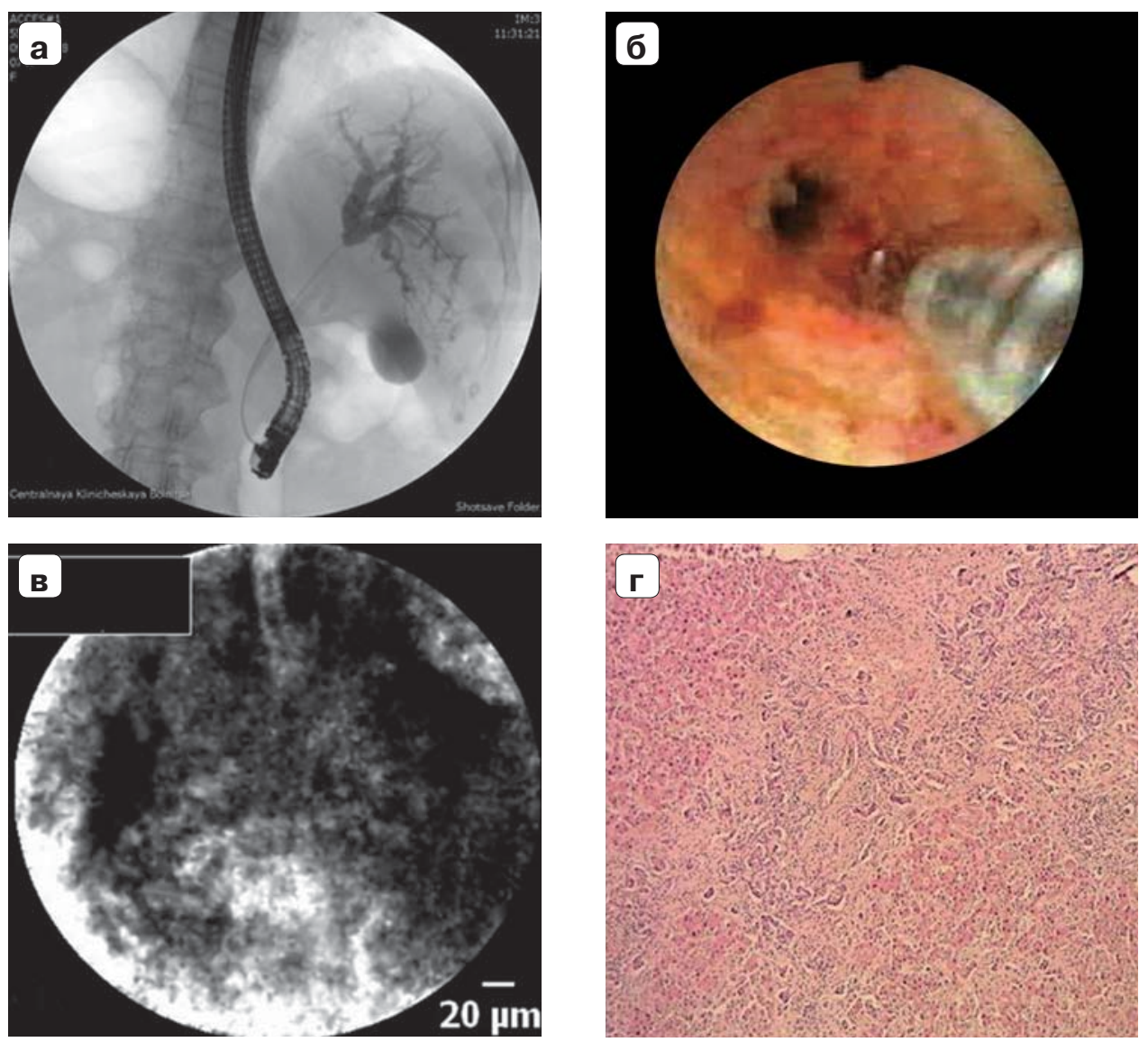

Рис. 2. Холангиокарцинома с поражением конфлюенса долевых протоков: а - холангиограмма, стриктура в зоне конфлюенса; б - эндофото, гиперемированная мелкобугристая слизистая в зоне стриктуры при холангиоскопии; в эндоскопическое микрофото, нарушение микроструктур при КЛЭМ; г - микрофото, гистологическая картина.

Fig. 2. Cholangiocarcinoma with lesion of lobar ducts confluence: a - cholangiogram, confluence stricture; b - endoscopic photo, hyperemic small-bumpy mucosa within the stricture area; $\mathrm{c}$ - endoscopic photo, CLM data of impaired microstructures; $\mathrm{d}-$ endoscopic photo, histological picture.

ный результат получен в 1 (3,6\%) наблюдении, истинно отрицательный - в 15 (53,6\%). Диагностическая значимость комбинации ЭРХПГ, холедохоскопии, КЛЭМ и биопсии в диагностике злокачественных стриктур ОЖП обусловлена высокой чувствительностью метода - 91,7\%, специфичностью - 93,7\%, прогностической ценностью положительного результата - 91,7\%, прогностической ценностью отрицательного результата - 93,7\%, общей точностью теста, составляющей $92,8 \%$.

В $1(3,6 \%)$ наблюдении развился острый отечный панкреатит, что не превышает частоту панкреатита при стандартной ЭРХПГ. Транзиторную амилаземию при отсутствии клинических проявлений панкреатита отмечали несколько чаще, чем при ЭРХПГ, - у 4 (14,3\%) пациентов.

\section{Обсуждение}

В последнее время отмечен рост числа пациентов со стриктурами внепеченочных желчных протоков. В результате повсеместного внедрения лапароскопической холецистэктомии, с одной стороны, возросла частота интраоперационного повреждения желчных протоков, в том числе в результате термовоздействия, проявляющегося отсроченным рубцовым стенозом. С другой стороны, по данным литературы, возросло число пациентов со злокачественными новообразованиями органов ГПДЗ, составляющими порядка 9\% всех злокачественных опухолей. Хирургическая тактика при стенозе доброкачественного и злокачественного генеза кардинально отличается: при опухолях это преимущественно радикальное хирургическое вмешательство, а при рубцовом стенозе методом выбора является миниинвазивное эндоскопическое или чрескожное вмешательство на желчных протоках. Поэтому ключевую роль в определении тактики лечения играет дифференциальная диагностика доброкачественного и злокачественного стеноза внепеченочных желчных протоков.

Традиционно применяемые методы лучевой диагностики (КТ, МРТ, УЗИ) не обладают достаточной информативностью. По данным отдельных авторов, частота не уточненных лучевыми методами заболеваний достигает $30 \%$ [2, 3]. Даже 
прямое контрастирование протоков при ЭРХПГ или ЧЧХС не всегда позволяет судить об этиологии стриктуры. Кроме того, ЭРХПГ относится к категории инвазивных вмешательств с высоким уровнем осложнений и может быть рекомендована или как лечебное, или с целью окончательной диагностики, поскольку позволяет получить материал для морфологической верификации. Однако неприцельная биопсия, проводимая под рентгеноскопическим контролем, сопровождается достаточно высоким уровнем ложноположительных и ложноотрицательных результатов. По данным ряда исследователей, браш-цитология, проводимая под рентгенологическим контролем, имеет чувствительность только 30-57\% [5-7]. Чувствительность метода в диагностике холангиокарциномы несколько больше, однако в приводимых данных можно отметить большой диапазон результатов от 20 до 70\%, что, вероятно, связано с небольшим числом наблюдений и опытом оператора [8, 9]. Данные исследований об эффективности получения гистологического материала под визуальным контролем противоречивы. Чувствительность биопсии даже под контролем зрения в диагностике природы стриктуры не превышает $49 \%$, достигая $66 \%$ при внутрипротоковой неоплазии $[10,11]$. Таким образом, дифференциальная диагностика стриктур ОЖП остается одной из наиболее актуальных проблем эндоскопии, а раннее обнаружение холангиокарциномы трудновыполнимо.

Новым методом в диагностике заболеваний внепеченочных желчных протоков является КЛЭМ, позволяющая прижизненно, в реальном времени оценить изменения стенки протока на микроструктурном уровне.

По данным литературы, метод позволяет увеличить диагностическую чувствительность до $91,7 \%$, специфичность - до $93,7 \%$ [7, 12, 13]. В 2010 г. проведен метаанализ, основанный на результатах 112 исследований. В большинстве публикаций число выполненных исследований в одной клинике составило 8-14.

В обсуждаемой работе на достаточно большом числе наблюдений показано, что, несмотря на трудоемкость, при определенном опыте оператора «выполнимость» метода высока и достигает 93,3\%. Метод, несмотря на свою техническую сложность, не ведет к увеличению уровня осложнений и соответственно не имеет противопоказаний, отличных от ЭРХПГ. Достигнутые чувствительность 91,7\%, специфичность 93,8\% и общая точность 92,8\%, сопоставимые с данными литературы, показывают, что эффективность холедохоскопии и КЛЭМ с прицельной биопсией значительно больше, чем ЭРХПГ с так называемой неприцельной биопсией.

\section{Заключение}

Применение комплекса современных эндоскопических методов сопровождается улучшением результатов диагностики, что позволяет выполнять этиологически оправданные вмешательства пациентам с заболеваниями внепеченочных желчных протоков и оптимизировать результаты лечения.

\section{Участие авторов}

Солодинина Е.Н. - дизайн статьи, редактирование, утверждение окончательного варианта.

Фомичева Н.В. - сбор и обработка материала, статистическая обработка данных, написание статьи.

Ульянов Д.Н. - статистическая обработка данных.

\section{Authors' participation}

Solodinina E.N. - article design, editing, approval of the final version.

Fomicheva N.V. - collection and processing of material, statistical analysis, writing text.

Ulyanov D.N. - statistical analysis.

\section{Список литературы}

1. Каприн А.Д., Старинский В.В., Петрова Г.В. Злокачественные новообразования в России в 2013 году (заболеваемость и смертность). М.: МНИОИ им. П.А. Герцена, 2015. $250 \mathrm{c}$.

2. Бобоев Б.Д. Роль эндоскопической ультрасонографии в диагностике холедохолитиаза и воспалительных стриктур желчных протоков. Вестник хирургии им. И.И. Грекова. 2012; 3: 39-41.

3. Бобоев Б.Д. Ультразвуковое исследование в диагностике желчнокаменной болезни и ее осложнений. Вестник хирургии им. И.И. Грекова. 2012; 171 (2): 21-24.

4. Шулешова А.Г., Завьялов М.О., Ульянов Д.Н. Возможности комбинированных эндоскопических методов диагностики с использованием зондовой конфокальной эндомикроскопии в выявлении неоплазий желудка. Хирургия. Журнал им. Н.И. Пирогова. 2014; 8: 9-15.

5. Boberg K.M., Jebsen P., Clausen O.P., Foss A., Aabakken L., Schrumpf E. Diagnostic benefit of biliary brush cytology in cholangiocarcinoma in primary sclerosing cholangitis. J. Hepatol. 2006; 45 (4): 568-574. https://doi.org/10.1016/j.jhep.2006.05.010.

6. Victor D.W., Sherman S., Karakan T. Current endoscopic approach to indeterminate biliary strictures. World J. Gastroenterol. 2012; 18 (43): 6197-6205.

https://doi.org/10.3748 / wjg.v18.i43.6197.

7. Siqueira E., Schoen R.E., Silverman W., Martini J., Rabinovitz M., Weissfeld J.L., Slivka A. Detecting cholangiocarcinoma in patients with primary sclerosing cholangitis. Gastrointest. Endosc. 2002; 56 (1): 40-47. https://doi.org/10.1067/mge.2002.125105.

8. Geraci G., Pisello F. Endoscopic cytology in biliary strictures. Personal experience. G. Chir. 2008; 29 (10): 403-406.

9. Smith I., Kline P.E., Gaidhane M. A review on the use of confocal laser endomicroscopy in the bile duct. Gastroenterol. Res. Pract. 2012; Volume 2012, Article ID 454717, 5 pages. https://doi.org/10.1155/2012/454717. 
10. Jong H.M., Hyun J.C. The role of direct peroral cholangioscopy using an ultraslim endoscope for biliary lesions: indications, limitations, and cjmplications. Clin. Endosc. 2013; 46 (5): 537-539. https://doi.org/10.5946 / ce.2013.46.5.537.

11. Lee T.Y., Cheon Y.K., Shim C.S. Photodynamic therapy in patients with advanced hilar cholangiocarcinoma: percutaneous cholangioscopic versus perorel transpapillary approach. Photomed. Laser Surg. 2016; 34 (4): 150-156. https://doi.org/10.5946 / ce.2013.46.1.38.

12. Meining A., Shah R.J., Slivka A., Pleskow D., Chuttani R., Stevens P.D., Becker V., Chen Y.K. Classification of probe-based confocal laser endomicroscopy findingins in pancreatobiliary stricture. Endoscopy. 2012; 44 (3): 251-257. https://doi.org/10.1055 / s-0031-1291545.

13. Steiner C.A., Bass E.B., Talamini M.A., Pitt H.A., Steinberg E.P. Surgical rates and operative mortality for open and laparoscopic cholecystectomy in Maryland. N. Engl. J. Med. 1994; 330 (6): 403-408.

\section{References}

1. Kaprin A.D., Starinskiy V.V., Petrova G.V. Zlokachestvennie novoobrazovaniya $v$ Rossii v 2013 godu (zabolevaemost $i$ smertnost) [Malignancies in Russia in 2013 (morbidity and mortality)]. Moscow: MNIOI im. P.A. Herzena, 2015. 250 p. (In Russian)

2. Boboev B.D. The role of endoscopic ultrasonography in the diagnosis of cholelithiasis and inflammatory strictures of the bile ducts. Vestnik khirurgii im. I.I. Grekova. 2012; 3: 39-41. (In Russian)

3. Boboev B.D. Ultrasound in the diagnosis of cholelithiasis and its complications. Vestnik khirurgii im. I.I. Grekova. 2012; 171 (2): 21-24. (In Russian)

4. Shuleshova A.G., Zavyalov M.O., Ulyanov D.N. Endoscopic diagnostic methods with confocal endomicroscopy in the diagnosis of gastric malignancies. Pirogov Russian Journal of Surgery $=$ Khirurgiya. Zhurnal imeni N.I. Pirogova. 2014; 8: 9-15. (In Russian)
5. Boberg K.M., Jebsen P., Clausen O.P., Foss A., Aabakken L., Schrumpf E. Diagnostic benefit of biliary brush cytology in cholangiocarcinoma in primary sclerosing cholangitis. J. Hepatol. 2006; 45 (4): 568-574. https://doi.org/10.1016/j.jhep.2006.05.010.

6. Victor D.W., Sherman S., Karakan T. Current endoscopic approach to indeterminate biliary strictures. World J. Gastroenterol. 2012; 18 (43): 6197-6205.

https://doi.org/10.3748 / wjg.v18.i43.6197.

7. Siqueira E., Schoen R.E., Silverman W., Martini J., Rabinovitz M., Weissfeld J.L., Slivka A. Detecting cholangiocarcinoma in patients with primary sclerosing cholangitis. Gastrointest. Endosc. 2002; 56 (1): 40-47. https://doi.org/10.1067/mge.2002.125105.

8. Geraci G., Pisello F. Endoscopic cytology in biliary strictures. Personal experience. G. Chir. 2008; 29 (10): 403-406.

9. Smith I., Kline P.E., Gaidhane M. A review on the use of confocal laser endomicroscopy in the bile duct. Gastroenterol. Res. Pract. 2012; Volume 2012, Article ID 454717, 5 pages. https://doi.org/10.1155/2012/454717.

10. Jong H.M., Hyun J.C. The role of direct peroral cholangioscopy using an ultraslim endoscope for biliary lesions: indications, limitations, and cjmplications. Clin. Endosc. 2013; 46 (5): 537-539. https://doi.org/10.5946 / ce.2013.46.5.537.

11. Lee T.Y., Cheon Y.K., Shim C.S. Photodynamic therapy in patients with advanced hilar cholangiocarcinoma: percutaneous cholangioscopic versus perorel transpapillary approach. Photomed. Laser Surg. 2016; 34 (4): 150-156. https://doi.org/10.5946 / ce.2013.46.1.38.

12. Meining A., Shah R.J., Slivka A., Pleskow D., Chuttani R., Stevens P.D., Becker V., Chen Y.K. Classification of probe-based confocal laser endomicroscopy findingins in pancreatobiliary stricture. Endoscopy. 2012; 44 (3): 251-257. https://doi.org/10.1055 / s-0031-1291545.

13. Steiner C.A., Bass E.B., Talamini M.A., Pitt H.A., Steinberg E.P. Surgical rates and operative mortality for open and laparoscopic cholecystectomy in Maryland. N. Engl. J. Med. 1994; 330 (6): 403-408.

\section{Сведения об авторах [Authors info]}

Солодинина Елена Николаевна - доктор мед. наук, заведующая эндоскопическим отделением ФГБУ “ЦКБ с поликлиникой” УДП РФ (orcid.org/0000-0002-5462-2388).

Фомичева Наталья Владимировна - врач-эндоскопист эндоскопического отделения ФГБУ “ЦКБ с поликлиникой” УДП РФ (orcid.org/0000-0003-3921-9934).

Ульянов Дмитрий Николаевич - канд. мед. наук, руководитель отделения эндоскопии EMC (orcid.org/0000-0001-68611201).

Для корреспонденщии *: Фомичева Наталья Владимировна - 121359, Москва, ул. Маршала Тимошенко, д. 15, Российская Федерация. Тел.: +7-903-592-24-64. E-mail: clarabobchik@gmail.com

Elena N. Solodinina - Doct. of Med. Sci., Head of the Endoscopic Department of Central Clinical Hospital with a Polyclinic of Presidential Administration of the Russian Federation (orcid.org/0000-0002-5462-2388).

Nataliya V. Fomicheva - Endoscopist of the Endoscopic Department of Central Clinical Hospital with a Polyclinic of Presidential Administration of the Russian Federation (orcid.org/0000-0003-3921-9934).

Dmitry N. Ulyanov - Cand. of Med. Sci., Head of the Endoscopic Department of European Medical Center, Russian Federation (orcid.org/0000-0001-6861-1201).

For correspondence *: Natalya V. Fomicheva - Endoscopic Department, Moscow Central Clinical Hospital with a Polyclinic of Presidential Administration of the Russian Federation; 15, Marshala Timoshenko str., Moscow, 121359, Russian Federation. Phone: +7-903-592-24-64. E-mail: clarabobchik@gmail.com

Статья поступила в редакцию журнала 15.02.2019.

Received 15 February 2019.
Принята к публикации 19.02.2019.

Accepted for publication 19 February 2019. 